\title{
Incidencia y evolución de membrana hialina en menores de 35 semanas según crecimiento intrauterino
}

\author{
PATRICIA MENA N..${ }^{1,2}$, JEANNE SMITH R. ${ }^{1}$, BEATRIZ MILET L. ${ }^{1}$, CLAUDIA TORO J. ${ }^{1,2}$, \\ FERNANDO ARREDONDO S. ${ }^{1}$, ADOLFO LLANOS M. ${ }^{3}$ \\ 1. Servicio de Recién Nacidos, CA Dr Sótero del Río. \\ 2. Sección Neonatología. División de Pediatría. Pontificia Universidad Católica de Chile.
}

\begin{abstract}
Incidence and evolution of hyaline membrane in newborns under 35 weeks of gestational age according intrauterine growth

Introduction: It is thought that intrauterine growth restriction induces respiratory maturation. The information varies if the studies consider analysis based on birth weight or gestational age. Objective: The goal of this study is to compare the incidence and evolution of hyaline membrane disease (HMD) between small and adequate premature babies under 35 weeks of gestational age $(<35$ wGA) based on data in the literature. Patients and Methods: Two databases were created and analyzed: a) 2022 newborns < 35 wGA admitted to the Service, whose incidence of HMD was calculated, and b) 733 newborns < 35 wGA with HMD and treated with surfactant, to describe the evolution. Results: Analysis of GA group shows a higher incidence of HMD (35.2\%) among small for GA, and less (29.1\%) among those who are not small for GA (p: 0.026). If a subset is formed for the newborns $<1500 \mathrm{~g}$ in brithweight, those small for gestational age have a lower incidence (47.5\%) than those adequate for GA (60.7\%). Logistic regression analysis for discharge with oxygen of newborns with HMD shows association with lower z score for birthweight, corticosteroid use and oxygen dependence at 36 weeks. Conclusions: Preterm newborns small for GA show a higher incidence of HMD and oxygen dependence when comparing for GA.
\end{abstract}

(Key words: Small for gestational age, hyaline membrane, bronchopulmonary displasia, intrauterine growth restriction, oxygen dependence).

Rev Chil Pediatr 2011; 82 (5): 395-401

\section{RESUMEN}

Introducción: Tradicionalmente se ha considerado que la restricción de crecimiento intrauterina produce maduración respiratoria, pero la información es diferente según si los estudios consideran el análisis por grupos de peso de nacimiento o edad gestacional. Objetivo: El objetivo de este análisis fue comparar la incidencia y evolución de membrana hialina, de los prematuros menores de 35 semanas de edad gestacional según fueran pequeños o no para edad gestacional. Pacientes y Método: Se analizaron dos bases de datos: 2022 menores de 35 semanas hospitalizados en el Servicio para determinar incidencia de membrana hialina y 733 menores

Trabajo recibido el 05 de enero de 2011, devuelto para corregir el 08 de febrero de 2011, segunda versión el 24 de febrero de 2011, aceptado para publicación el 09 de mayo de 2011.

Correspondencia a:

Patricia Mena N.

E-Mail: pmenanani@gmail.com 
de 35 semanas tratados con surfactante con diagnóstico de membrana hialina para comparar evolución de ésta. Resultados: El análisis por grupos de edad gestacional muestra una incidencia de membrana hialina mayor, de $35,2 \%$, en los pequeños para la edad gestacional, y de $29,1 \%$ en los no pequeños (p: 0,026). Si se analiza sólo menores de 1500 gramos de peso de nacimiento, el grupo pequeño tiene una incidencia menor, de 47,5\%, y los no pequeños de 60,7\%. El análisis de regresión logística para alta con oxígeno de los que tuvieron membrana hialina, muestra asociación con menor puntaje z de peso de nacimiento, uso de corticoides y dependencia de oxígeno a las 36 semanas. Conclusiones: El recién nacido pretérmino pequeño para edad gestacional tiene mayor incidencia de membrana hialina y evoluciona con mayor dependencia de oxígeno al comparar por edad gestacional.

(Palabras clave: Pequeño para edad gestacional, membrana hialina, displasia broncopulmonar, restricción de crecimiento intrauterino, dependencia de oxígeno).

Rev Chil Pediatr 2011; 82 (5): 395-401

\section{Introducción}

Cuando se anuncia el nacimiento de un recién nacido prematuro, que además tiene una restricción del crecimiento intrauterino (RCIU) se tiende a pensar, tanto en el ambiente obstétrico, como neonatal, que su patología neonatal respiratoria será menor, dada la información que el stress fetal favorece la aceleración de la maduración pulmonar ${ }^{1,2}$.

Estudios epidemiológicos, considerando el bajo peso para edad gestacional como un índice de RCIU, reportan resultados diferentes ${ }^{3-8}$. Los grupos analizados más frecuentemente son grupos seleccionados por peso, por ejemplo los menores de 1500 gramos o los menores de 1000 gramos de peso de nacimiento, que constituye una diferente población en estudio que si se analiza los menores de 34 semanas o de 30 semanas de edad gestacional, por lo que, se puede esperar que sus resultados sean diferentes ${ }^{3}$.

Un análisis en una población seleccionada por peso de nacimiento, por ejemplo, la población de menores de $1500 \mathrm{~g}$ al nacer, claramente muestra menor riesgo respiratorio en los niños con RCIU o los pequeños para edad gestacional (PEG), pero este grupo tiene mayor edad gestacional ${ }^{4,5}$. Análisis más recientes muestran que agrupados por grupos de edad gestacional, los PEG presentan un riesgo igual o mayor de problemas respiratorios, especialmente entre los menores de 30 semanas de edad gestacional ${ }^{6-8}$.

Aclarar esta información es importante para caracterizar riesgos y considerar medidas preventivas y terapéuticas.

El objetivo de este análisis fue comparar la incidencia y evolución de membrana hialina, de los prematuros menores de 35 semanas de edad gestacional según fueran pequeños o no para edad gestacional dada la diferente información existente en la literatura.

\section{Pacientes y Método}

Se analizó la base de datos de 2198 egresados en el Servicio de Recién Nacidos del Hospital Dr. Sótero del Río, entre el 2000 y 2008 menores de 35 semanas de gestación hospitalizados al nacer con la que se determina la incidencia de membrana hialina y letalidad de los grupos que se estudiaron Se excluyeron los casos con traslado precoz del Servicio, por no contar con información completa sobre su hospitalización (176 casos, 7,9\%) (Base 1). Además se analizaron los datos de 733 menores de 35 semanas registrados en el Programa de Surfactante del Ministerio de Salud, de nuestro hospital, que incluye todos los pacientes con diagnóstico de enfermedad de membrana hialina que recibieron surfactante (Base 2) entre 1998 y 2008, exceptuando gran parte de 2003, cuyos datos no fueron reunidos. Esta base contenía información sobre corticoides prenatales, número de dosis de surfactante, edad de uso de éste y patologías relacionadas que no estaba registrada en la Base 1 . Con esta base se analizó la evolución aguda y crónica de los niños con membrana hialina que requirieron surfactante. Se realizó la clasificación de crecimiento intrauterino para cada caso según peso para edad gestacional en semanas cumplidas según curva de Pittaluga ${ }^{9}$. La edad gestacional fue estimada por fecha de última regla, confirmada 
MEMBRANA HIALINA

por ecografía y por examen clínico pediátrico. En los casos de antecedentes no concordantes o inexistentes se realizó evaluación pediátrica con el test de Ballard ${ }^{10}$. Los casos con peso menor al percentil 10 fueron clasificados como pequeños para edad gestacional (PEG). Los casos con peso sobre el percentil 90 fueron clasificados como grandes para edad gestacional (GEG). Los con peso igual o mayor que el percentil 10 o igual o menor que el percentil 90 fueron clasificados como adecuados para edad gestacional (AEG).

Como el grupo de GEG constituyó sólo el $4,7 \%$ en la Base 1 y el 3,9\% en la Base 2, se decidió establecer la comparación entre los PEG y los que no eran PEG, ya sea AEG o GEG (No PEG). Además se determinó el puntaje z de peso para edad gestacional en los casos que recibieron surfactante (base 2).

\section{Análisis estadístico}

Se revisaron los datos en una planilla Excel y se analizaron utilizando programa estadístico EPI Info. Se compararon los datos con $\chi^{2}$ o test de Fisher, para variables discontinuas o categoricas y anova para variables continuas, con test Kruskal Wallis para distribuciones no normales, considerándose significativo un $\mathrm{p}$ menor de 0,05 . Se analizaron razón de riesgo y sus límites para las variables estudiadas según sean PEG o No PEG. Se realizó análisis de regresión logística para evaluar factores asociados a la patología respiratoria crónica con alta con oxígeno a domicilio del grupo con enfermedad de membrana hialina.

\section{Resultados}

El análisis de 2022 casos de Base 1 mostró una incidencia global del diagnóstico de Membrana Hialina de 30,6\%, una letalidad global de $11 \%$ y por membrana hialina de $18,9 \%$.

La distribución de la población como No PEG y PEG se muestra en la tabla 1; se observa: la letalidad general, por membrana hialina, la incidencia de $\mathrm{MH}$ y el uso de surfactante, mayores en los PEG, todos estadísticamente significativos.

Se realizó un análisis de incidencia de membrana hialina según sean PEG o NO PEG $\mathrm{y}$ por semanas de edad gestacional, que se muestra en la figura 1 . Puede observarse que la incidencia de membrana hialina $(\mathrm{MH})$ es similar, con una leve mayor incidencia en los PEG en la población total, que es estadísticamente significativa pero sólo con una razón de riesgo (RR) de 1,09 (IC 1,01-1,17), o sea un PEG tiene un 9\% de mayor riesgo que un No PEG

Tabla 1. Hospitalizados menores de $\mathbf{3 5}$ semanas según peso para edad gestacional

\begin{tabular}{|lccc|}
\hline & No PEG & PEG & p \\
\hline $\mathrm{n}$ & 1516 & 506 & \\
\hline$\%$ & 75 & 25 & \\
\hline Letalidad \% & 9,8 & 16,2 & 0,000 \\
\hline Incidencia $\mathrm{MH} \%$ & 29,1 & 35,2 & 0,026 \\
\hline Letalidad por $\mathrm{MH} \%$ & 16,3 & 25,3 & 0,0059 \\
\hline Uso surfactante \% & 24,7 & 34,6 & 0,0000 \\
\hline
\end{tabular}

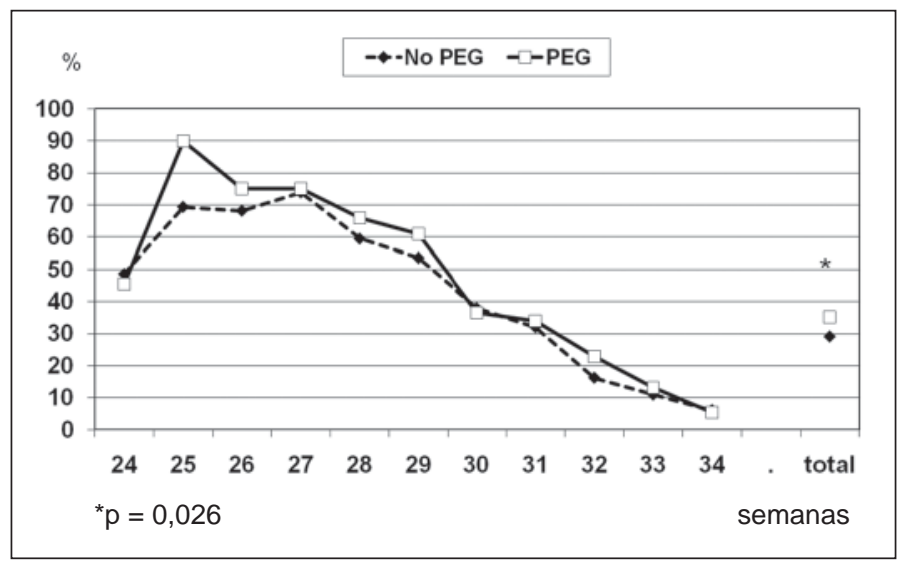

Figura 1. Incidencia de $\mathrm{MH}$ por semanas de edad gestacional en PEG y No PEG. Por edad gestacional no hay diferencias estadísticamente significativa ( $\chi^{2}$ o prueba Fisher). 
Tabla 2. Incidencia de MH considerando grupos por peso o por edad gestacional

\begin{tabular}{|lcccccc|}
\hline & $\mathbf{n}$ & \% MH no PEG & \% MH PEG & RR & Límites IC & P \\
\hline Peso nacimiento $<1500 \mathrm{~g}$ & 809 & 60,7 & 47,5 & 0,75 & $0,64-0,87$ & 0,000 \\
\hline Edad gestacional $<32 \mathrm{sem}$ & 957 & 49,9 & 57,6 & 1,18 & $1,00-1,38$ & 0,017 \\
\hline
\end{tabular}

de presentar membrana hialina, esto es para el total de menores de 35 semanas nacidos en el período observado.

En la tabla 2 se muestra la incidencia de MH cuando se selecciona la población de menores de 1500 gramos de peso de nacimiento o la de menos de 32 semanas de edad gestacional. Los resultados son claramente diferentes: para un grupo seleccionado por peso de nacimiento, el riesgo de membrana hialina es claramente mayor en los No PEG, pero, si se selecciona un grupo por edad gestacional, hay un mayor riesgo del PEG.

El análisis de la base 2, de 733 menores de 35 semanas tratados con surfactante, entre 1998 y 2008, se observa en la tabla 3, que muestra mayor letalidad en los PEG; éstos recibieron más corticoides prenatales, tuvie-

Tabla 3. Casos tratados con surfactante

\begin{tabular}{|c|c|c|c|}
\hline & No PEG & PEG & $\mathbf{p}$ \\
\hline $\mathrm{n}$ & 537 & 196 & \\
\hline Letalidad \% & 19,2 & 28,6 & 0,0037 \\
\hline Peso $\mathrm{g}^{*}$ & $1323(463)$ & 950 (328) & 0,000 \\
\hline $\begin{array}{l}\text { Edad gestacional* } \\
\text { semanas }\end{array}$ & $28,5 \quad(2,6)$ & $28,8 \quad(2,6)$ & ns \\
\hline Corticoides \% & 80,1 & 89,1 & 0,002 \\
\hline Cesárea \% & 69 & 93,4 & 0,000 \\
\hline Sexo femenino \% & 40,9 & 47,7 & ns \\
\hline Neumotórax \% & 8,9 & 7,6 & ns \\
\hline Ventilación días* & $\begin{array}{l}8,1(14,4) \\
2\end{array}$ & $3^{9,1(16,7)}$ & ns \\
\hline $\begin{array}{l}\text { Oxígenoterapia } \\
\text { días* }\end{array}$ & $\begin{array}{l}33,6 \\
11\end{array}$ & $\begin{array}{l}37,2 \\
13\end{array}$ & ns \\
\hline $\begin{array}{l}\text { Hospitalización } \\
\text { días* }\end{array}$ & $\begin{array}{l}55,8 \\
48,5\end{array}$ & $\begin{array}{l}62,9 \\
48\end{array}$ & ns \\
\hline $\begin{array}{l}\text { Edad primera dosis* } \\
\text { horas }\end{array}$ & $\begin{array}{l}3,4 \\
2\end{array}$ & $\begin{array}{l}4,5 \\
2\end{array}$ & 0,03 \\
\hline $\begin{array}{l}\text { Edad segunda } \\
\text { dosis* horas }\end{array}$ & $\begin{array}{c}10,3 \\
8\end{array}$ & $\begin{array}{r}12,1 \\
9,1\end{array}$ & ns \\
\hline
\end{tabular}

*promedio (desviación estándar) mediana. ron menos peso de nacimiento y similar edad gestacional que los No PEG. La incidencia de complicaciones como neumotórax, hemorragia pulmonar y hemorragia intracraneana grave (III y IV) fue similar.

En el grupo que egresa vivo (tabla 4), sólo se observa una diferencia significativa en el \% de alta con oxígeno que es mayor en el grupo PEG, no habiendo diferencias estadísticamente significativas en la duración de la oxígenoterapia ni de la ventilación asistida, pero con cifras coherentes de mayor compromiso respiratorio en los $\mathrm{PEG}$.

Los menores de 32 semanas que son PEG presentaron mayores días de oxígenoterapia, de hospitalización y un porcentaje mayor se va de alta con oxígeno. Cuando se analizó el grupo de menos de 30 semanas al nacer, estas diferencias son aún más marcadas. Así el 46,6\% de los PEG, menor de 30 semanas, tratado con surfactante, egresó con oxígeno domiciliario, comparado con el 27,1\% del No PEG.

No hubo diferencias en el número de dosis de surfactante, que recibieron los PEG y los No PEG: alrededor del $44 \%$ de los casos recibió una, el $44 \%$, dos, el $10 \%$, tres y el $2 \%$, cuatro dosis de surfactante.

Tabla 4. Casos tratados con surfactante, egresados vivos

\begin{tabular}{|llll|}
\hline & No PEG & \multicolumn{1}{c|}{ PEG } & p \\
\hline DBP a los 28 días \% & 46,8 & 49,3 & ns \\
\hline DBP a las 36 semanas \% & 37,5 & 40,7 & ns \\
\hline Ventilación días* & $7,9(13,6)$ & $9,4(17,3)$ & ns \\
\hline Oxígenoterapia días* & 2 & 3 & \\
& $38,0(40)$ & $46,5(47)$ & ns \\
Hospitalización días* & 18,5 & 27,5 & \\
& $65,9(56,2)$ & $70,8(39)$ & ns \\
Alta con oxígeno \% & 14,7 & 62,5 & \\
\hline
\end{tabular}

*promedio (desviación estándar).

mediana. 
Tabla 5. Modelo de regresión logística de factores predictores de alta con $\mathrm{O}_{2}$, de los tratados con surfactante

\begin{tabular}{|llll|}
\hline Factores predictores & OR & P & 95\% IC \\
\hline $\begin{array}{l}\text { Variación }+0,25 \\
\text { z-score peso/edad gestacional }\end{array}$ & 0,9 & 0,02 & $0,85-0,97$ \\
\hline Uso de corticoides prenatal \% & 2,25 & 0,04 & $1,03-4,8$ \\
\hline DBP a los 28 días \% & 1,1 & 0,8 & $0,4-2,9$ \\
\hline DBP a las 36 semanas \% & 4,3 & 0,002 & $1,7-11,0$ \\
\hline Ventilación > mediana 3 días & 1,7 & 0,06 & $0,97-3,1$ \\
\hline Edad post-concepcional al alta & 1,0 & 0,25 & 1,1 \\
\hline
\end{tabular}

En el análisis de regresión logística para alta con oxígeno, considerando los egresados vivos, los factores significativamente asociados fueron la dependencia de oxígeno a las 36 semanas, los corticoides prenatales y el tener menor puntaje z para la edad gestacional (tabla 5).

\section{Discusión}

Los datos muestran que los PEG, comparados por edad gestacional, con los No PEG, tuvieron igual o mayor incidencia de membrana hialina y la evolución de los niños con membrana hialina tuvo una tendencia a más prolongados requerimientos de oxígeno, lo que coincide con otros análisis similares ${ }^{6,11,13-18}$. La información de mayor maduración respiratoria de los niños con RCIU se origina en una época en que la duración de la gestación no era rutinariamente evaluada con ecografía precoz y la comparación se realizaba por grupos de peso menor a cierta cifra, como 1500 o 2000 gramos, donde los PEG eran de mayor edad gestacional, lo que explica su mayor madurez. La información publicada para grupos de menos de 32 semanas es muy clara en ese sentido, y el riesgo de mayor letalidad y de complicaciones respiratorias está aumentado en los PEG, aún corrigiendo para uso de corticoides, sexo y tipo de parto ${ }^{11}$, no hay evidencias de un efecto diferente de los corticoides prenatales por situación de crecimiento intrauterino ${ }^{11}$. En otro análisis retrospectivo no encuentran diferencias significativas en la incidencia de SDR en PEG o niños con restricción de crecimiento, hayan o no recibido corticoides prenatales, pero se menciona la necesidad de un estudio controlado, randomizado al respecto ${ }^{12}$.

En 1991, Arnold ya advierte lo inadecuado que puede ser la evaluación de menores de 1500 g de peso al nacer en aspectos relacionados con crecimiento y madurez, considerando la diferente edad gestacional que pueden tener, y lo más apropiado que es comparar por grupos de edad gestacional ${ }^{3}$.

Los siguientes trabajos, en que comparan PEG y AEG por grupo de edad gestacional son en general coincidentes: El análisis de datos de una gran población de nivel socioeconómico bajo en Texas, muestra claramente el mayor riesgo de SDR en los PEG para una misma edad gestacional, aun controlando por sexo y $\mathrm{raza}^{6}$. La magnitud del mayor riesgo del PEG es similar a la que tiene el sexo masculino y la raza blanca.

En análisis de regresión logística, en 2700 casos sin malformaciones entre 24 y 31 semanas, menores de 1500 g, los PEG tuvieron mayor mortalidad, DBP y ROP ${ }^{5}$; también es diferente la distribución por sexo (más hombres AEG), más cesáreas en PEG, menos antecedentes obstétricos, salvo hipertensión, en el PEG ${ }^{5}$.

Otra evaluación de menores de 27 semanas claramente muestra igual SDR, pero mayor DBP, mayor ventilación mecánica, mayor ROP, en una muestra de 191 niños ${ }^{13}$.

En la red neonatal noruega de menores de 28 semanas o menores de 1000 g, con PEG definidos bajo el percentil 5, encontraron mayor DBP. Entre 22 y 25 semanas se describe mayor ECN y entre 26 y 27 semanas, mayor mortalidad $^{14}$. En este artículo el riesgo de pulmón crónico o dependencia de oxígeno a las 36 semanas, es significativamente mayor en pacientes con antecedentes de alteración de los flujos doppler en vasos umbilicales que en el que no tiene alteraciones ${ }^{14}$.

Análisis de un estudio multicéntrico de surfactante precoz y tardío muestra en el PEG mayor uso de Cpap, oxígenoterapia, mortalidad, retinopatía, duración de hospitalización y uso de dexametasona por DBP en niños entre 27 y 32 semanas $^{15}$. 
Mc Intire realiza un interesante análisis en una gran población de menores de 36 semanas evaluando la incidencia de SDR y la letalidad por grupos de percentil de peso, mostrando un aumento continuo a menor percentil, pero también un importante aumento en los niños con peso bajo el p 20 para la edad gestacional, especialmente entre los menores de 32 semanas ${ }^{16}$. En el mismo sentido, un análisis de riesgo en prematuros clasificados en 6 grupos de peso para edad gestacional muestra mucho mayor riesgo de DPB en el PEG, y una relación inversa entre peso para edad gestacional y riesgo de DBP. En el caso de mortalidad la diferencia es significativa cuando se ajusta por edad gestacional, sexo, uso de corticoides prenatales, embarazo múltiple y traslado prenatal, hasta el p25 ${ }^{17}$.

Entre menores de 37 semanas, hospitalizados, ser PEG da mayor mortalidad, duración de hospitalización y pulmón crónico. El SDR tiene menor frecuencia en PEG mayor o igual a 33 semanas $^{18}$. En nuestro estudio también se muestra una tendencia, en el mayor de 33 semanas, de una menor incidencia de SDR en los PEG que en los No PEG.

En 29916 menores de 35 semanas, comparan RCIU de diagnóstico prenatal, PEG y un grupo con los dos antecedentes o alguno de ellos. Encuentran mayor mortalidad, soporte respiratorio a los 28 días, ECN y ROP en los PEG o RCIU, aun ajustando por sexo, parto, corticoides y edad gestacional. En este análisis no se refieren a incidencia de síndrome de dificultad respiratoria ${ }^{19}$.

En menores de 33 semanas, Lay encuentra mayor SDR con edad gestacional de 25 a 28 semanas, pero menor en los mayores de 29 semanas y los corticoides prenatales tienen menos efecto en los PEG que en los $\mathrm{AEG}^{20}$.

La incidencia de SDR entre los nacidos entre 27 y 32 semanas es mayor en los PEG. Otro estudio no muestra lo mismo: de 27 a 35 semanas encuentran menor incidencia de SDR entre los $\mathrm{PEG}^{21}$.

Costa también encuentra mayor riesgo de DBP, duración de oxígenoterapia, hospitalización y ventilación asistida en los PEG ${ }^{22}$.

Cuando el análisis de los PEG se limita a los menores de $1500 \mathrm{~g}$, la mortalidad sigue siendo mayor en los PEG, pero tienden a tener menos SDR, y no hay diferencia en lesiones del SNC como hemorragia o leucomalasia periventricular. En otro análisis de menores de 1500 g el ser PEG significó menor riesgo de DBP, pero los PEG que presentaron DBP, tuvieron más prolongados requerimientos de oxígeno, todos requirieron oxígeno después de las 36 semanas de edad postconcepcional ${ }^{23}$.

Otro estudio de menores o igual a 32 semanas muestra mayor incidencia de DBP en los PEG y menor en los GEG, aun controlando por edad gestacional ${ }^{24}$.

La mayor incidencia de problemas respiratorios crónicos en los PEG podría estar relacionado con el compromiso del desarrollo vascular pulmonar, producido por insuficientes factores angiogénicos ${ }^{25}$. Similar mecanismo produce el aumento de incidencia de retinopatía del prematuro en el $\mathrm{PEG}^{26}$. La reciente descripción de mayor riesgo de DBP, con un Odd ratio de 18, cuando se controla por edad gestacional, puntaje $\mathrm{z}$ del peso de nacimiento y corioamnionitis en hijos de madres con preclampsia, apunta al mismo mecanismo dado el aumento de los factores antiangiogénicos en la preclampsia ${ }^{27}$.

Los prematuros de menos de 32 semanas, PEG, presentan mayor o igual membrana hialina al comparar por edad gestacional, pero están en mayor riesgo de problemas pulmonares crónicos que los AEG a toda edad gestacional, por lo cual, en los PEG debe considerarse en forma especial evitar factores agravantes de problemas respiratorios.

El concepto de que la restricción del crecimiento intrauterino acelera la maduración pulmonar y mejora el pronóstico respiratorio no se sustenta con los datos actuales, comparando por similar edad gestacional, en especial para los menores de 32 semanas.

\section{Referencias}

1.- Usher R: Clinical and therapeutic aspects of fetal malnutrition. Pediatr Clin North Am 1970; 17: 178.

2.- Gluck L, Kulovich M: Lecitin/sphingomyelin ratios in amniotic fluid in normal and abnormal pregnancy. Am J Obstet Gynecol 1975; 115: 539-46.

3.- Arnold CC, Kramer MS, Hobbs CA, McLean FH, Usher 
$R H$ : Very low birth weight: a problematic cohort for epidemiologic studies of very small or immature infants. Am J Epidemiol 1991; 134: 604-13.

4.- Bartels DB, Kreienbrock L, Dammann O, Wenzlaff $P$, Poets CF: Population based study on the outcome of small for gestational age newborns. Arch Dis Child Fetal Neonatal Ed. 2005; 90: F53-9.

5.- Regev RH, Lusky A, Dolfin T, et al: Excess mortality and morbidity among small-forgestational-age preterm infants: a population-based study. J Pediatr 2003; 143: 186-91.

6.- Tyson JE, Kennedy K, Broyles S, Rosenfeld CR: The small for gestational jage infant: accelerated or delayed pulmonary maturation? Increased or decreased survival? Pediatrics 1995; 95: 534-8.

7.- $\quad$ Thompson PJ, Greenough A, Gamsu HR, et al: Ventilatory requirements for respiratory distress syndrome in small-for-gestational-age infants. Eur J Pediatr 1992; 151: 528-31.

8.- Piper JM, Xenakis EM, McFarland M, Elliott BD, Berkus $M D$, Langer $O$ : Do growth retarded premature infants have different rates of perinatal morbidity and mortality than appropriately grown premature infants? Obstet Gynecol 1996; 87: 169-74.

9.- Pittaluga PE, Díaz AV, Mena NP, et al: Curva de crecimiento intrauterino para prematuros entre 23 a 36 semanas de edad gestacional. Rev Chil Pediatr 2002; 73: 135-41.

10.- Ballard JL, Khoury JC, Wedig K, Wang L, et al: New Ballard Score, expanded to include extremely premature infants. J Pediatr 1991; 119: 417-23.

11.- Bernstein IM, Horbar JD, Badger GJ, Ohlsson A, Golan A: Morbidity and mortality among very-low-birthweight neonates with intrauterine growth restriction. The Vermont Oxford Network. Am J Obstet Gynecol 2000; 182: 198-206.

12.- Torrance HL, Derks JB, Scherjon SA, et al: Is antenatal steroid treatment effective in preterm IUGR fetuses? Acta Obstet Gynecol 2009; 88: 1068-73.

13.- Bardin C, Zelkowitz P, Papageorgiou A: Outcome of small-forgestational age and appropriate-for-gestational age infants born before 27 weeks of gestation. Pediatrics 1997; 100 (2): e4.

14.- Westby Wold SH, Sommerfelt K, Reigstad H, Rønnestad $A$, et al: Neonatal mortality and morbidity in extremely preterm small for gestational age infants: a population based study Arch. Dis. Child. Fetal Neonatal Ed 2009; 94: F363-7.

15.- Gortner L, Wauer R, Stock GJ, et al: Neonatal outcome in small for gestational age infants: do they really better? J Perinat 1999; 27: 484-9.

16.- Zeitlin J, El Ayoubi M, Jarreau P, Draper E, et al: Impact of Fetal Growth Restriction on Mortality and Morbidity in a Very Preterm Birth Cohort. J Pediatr 2010; 157: 733-9.

17.- McIntire DD, Bloom SL, Casey BM, Leveno KJ: Birth Weight in relation to morbidity and mortality among newborn infants. N Eng J Med 1999; 340: 1234-8.

18.- Sharma P, McKay K, Rosenkrantz TS, et al: Comparisons of mortality and predischarge respiratory outcomes in small-for-gestational-age and appropriate-for-gestational-age premature infants. BMC Pediatr 2004; 4: 9.

19.- Garite TJ, Reese C, Thorp JA: Intrauterine growth restriction increases morbidity and mortality among premature neonates. Am J Obstet Gynecol 2004; 191: 481-7.

20.- Ley D, Wide-Swensson D, Lindroth $M$, et al: Respiratory distress syndrome in infants with impaired intrauterine growth. Acta Paediatr 1997; 86: 1090-6.

21.- Simchen MJ, Beiner ME, Strauss-Liviathan $N$, et al: Neonatal outcome in growth restricted versus appropriately grown preterm infants. Am J Perinatol 2000; 17: 187-92.

22.- Costa S, Maggio L, Sindico P, Cota F, De Carolis MP, Romagnoli C: Preterm Small for Gestational Age Infants Are Not at Higher Risk for Parenteral NutritionAssociated Cholestasis J Pediatr 2010; 156: 575-9.

23.- Korhonen P, Tammela O, Koivisto AM, et al: Frequency and risk factors in bronchopulmonary dysplasia in a cohort of very low birth weight infants. Early Hum Dev 1999; 54: 245-58.

24.- Lal MK, Bradley NM, Draper ES, et al: Chronic lung disease of prematurity and intrauterine growth retardation. A population-based study. Pediatrics 2003; 111: 483-7.

25.- Thebaud B, Lacaze-Masmonteil T: If Your Placenta Doesn't Have It, Chances Are Your Lungs Don’t Have It Either: The "Vascular Hypothesis" of Bronchopulmonary Dysplasia Starts In Utero J Pediatr 2010; 156: 521-3.

26.- Dhaliwal CA, Fleck BW, Wright E, Graham C, McIntosh $N$ : Retinopathy of prematurity in small-for-gestational age infants compared with those of appropriate size for gestational age. Arch Dis Child Fetal Neonatal Ed 2009; 94: F193-5.

27.- Hansen A, Barna C, Folkman J, McElrath T: Maternal Preeclampsia Predicts the Development of Bronchopulmonary Dysplasia J Pediatr 2010; 156: 532-6. 